Rev. Adm. Saúde - Vol. 18, № 72, jul. - set. 2018

http://dx.doi.org/10.23973/ras.72.116

ARTIGO ORIGINAL

\title{
Simulação e teoria das filas aplicadas na análise dos encaminhamentos de pacientes SUS para a especialidade médica de angiologia
}

Simulation and queuing theory applied in the analysis of the referrals of SUS patients to speciality of medical angiology

\section{Raphael Kaeriyama Silva ${ }^{1}$, Eric Magalhães Moraes ${ }^{2}$, Nancy Val y Val Peres da Mota ${ }^{3}$, Rodrigo Gouvea Mourad ${ }^{4}$}

1. Médico. Médico residente do Programa de Estudos Avançados em Administração Hospitalar e de Sistemas de Saúde (PROAHSA) do Hospital das Clínicas da Faculdade de Medicina da USP (HCFMUSP), São Paulo SP

2. Médico. Médico residente pelo PROAHSA do HCFMUSP, São Paulo SP

3. Médica especialista em administração hospitalar e sistemas de saúde. Membro do corpo técnico do PROAHSA do HCFMUSP, São Paulo SP

4. Engenheiro de produção mecânica especialista em modelos determinísticos aplicados à pesquisa operacional. São Paulo SP

\section{RESUMO}

O objetivo central deste trabalho consiste em abordar a problemática das filas de encaminhamentos médicos do SUS através de uma análise baseada na teoria das filas. A fim de propor soluções para tal problema, foi construído um modelo de simulação no software SIMUL8 Professional que contém, dentre outras, as informações do processo de chegada do paciente à fila e da capacidade de atendimento médico. Para o processo de chegada foram levantados os dados históricos da formação da fila incluindo a taxa de absenteísmo e para o processo de atendimento foram considerados: agenda média, distribuição dos tempos de consultas e outros fatores. Visto que o sistema se encontra instável, ou seja, a demanda supera a capacidade, há formação de fila. Através da técnica da simulação probabilística utilizada com o SIMUL8 observamos que o parâmetro do problema que mais impacta na otimização do fenômeno em questão é a taxa de atendimento médico. 
Palavras-chave: unidade básica de saúde, teoria de filas, simulação, referência

\begin{abstract}
The central objective of this work is to address the problem of the medical referral queues of SUS through an analysis based on the Theory of Queues. In order to propose solutions to this problem, a simulation model was built in the SIMUL8 Professional software, which includes, among others, the information about the patient's arrival process and the medical care capacity. For the arrival process, we considered the historical data of the queue formation including the absenteeism rate and for the process of attendance, the average agenda, distribution of doctor's appointment periods, unavailability and other factors. Since the system is unstable, that is, demand exceeds capacity, there is queuing. Using the probabilistic simulation technique in SIMUL8, we observed that the parameter of the problem that most impacts the optimization of the phenomenon in question is the attendance rate.
\end{abstract}

Keywords: health centers, systems theory, simulation, referral.

\title{
INTRODUÇÃO
}

As Unidades Básicas de Saúde (UBS) foram criadas no Brasil para serem as portas de entrada do Sistema Único de Saúde (SUS). É através da UBS que o cidadão recebe seu primeiro atendimento médico na rede pública (1), e a partir dela, pode ser encaminhado para serviços especializados estaduais, os quais são realizados pelos Ambulatórios Médicos de Especialidades (AME). Em 2013, a Prefeitura de São Paulo criou a rede de Hospitais da Rede Hora Certa $(\mathrm{HRHC})$, que também disponibiliza serviços de saúde especializados como alternativa de atendimento médico. Oferecem consultas médicas com especialistas, e são responsáveis por alguns exames solicitados por esses profissionais e por alguns procedimentos cirúrgicos eletivos.

Segundo a literatura, cerca de $80 \%$ das afecções de saúde populacional deveriam ser sanadas e acompanhadas pela Atenção Básica de Saúde, e apenas $20 \%$ delas seriam de responsabilidade do tratamento especializado (2). Após receber o atendimento inicial com o médico generalista na UBS, o paciente possui duas vias de conduta: ou mantém o acompanhamento na própria UBS, ou é encaminhado para avaliação e condução em um centro especializado. Caso haja a necessidade de avaliação do especialista, o paciente pode ter sua consulta agendada pelo sistema SIGA (Sistema Integrado de Gestão Ambulatorial) para a referência regional; pela Regulação Central Municipal a qual é responsável pelo agendamento direto em unidades especializadas diferentes daquela referenciada pela UBS de origem; ou pelo Sistema CROSS (Central de Regulação de Ofertas de Serviços de Saúde), que 
gera uma fila de âmbito estadual para os casos que não possuem vaga direta. Dessa forma, caso a unidade especializada de referência não possua vaga, o paciente deve aguardar na fila do SIGA ou do CROSS até o contato telefônico da UBS, a qual irá informar a data e horário do agendamento médico. Essa fila varia por especialidade e dependendo do tempo de espera pode comprometer a saúde do paciente e alterar seu prognóstico.

No caso da UBS analisada pelo presente estudo, localizada na cidade de São Paulo, observamos a presença de filas em praticamente todas as especialidades para as quais os pacientes são encaminhados, sendo uma das maiores a fila de encaminhamento para a especialidade médica de angiologia, a qual será o foco desse estudo. Outros exemplos de especialidades com fila encontrados nessa UBS são: Cardiologia, Neurologia, Ortopedia e Dermatologia. Isso leva os pacientes a ficarem meses aguardando um atendimento com o especialista, o que pode prejudicar a condução do cuidado de sua saúde. A demora por atendimento, dessa forma, pode ser consequência tanto da taxa elevada de encaminhamento médico do serviço de origem quanto da impossibilidade de absorção da demanda pelo $\mathrm{HRHC}$ de referência. Os encaminhamentos médicos devem ser pautados em justificativas clínicas plausíveis as quais demonstram uma necessidade real de avaliação especializada. Observa-se que muitas vezes os encaminhamentos são realizados erroneamente sendo que o caso poderia ser acompanhado na própria UBS, o que gera um fluxo demasiado de pacientes para o HRHC. No caso da cardiologia nessa UBS, por exemplo, $70 \%$ dos encaminhamentos por hipertensão arterial sistêmica são de casos leves, os quais poderiam ser tratados pelo médico generalista. Além disso, muitas vezes os pacientes acompanhados no serviço especializado demoram para receber alta médica e serem contrarreferenciados à UBS de origem, uma vez que não existe, no sistema, campo de agendamento de retorno do paciente à UBS após consulta no HRHC. Há uma inexistência de intercomunicação entre o serviço especializado e a UBS e há uma resistência tanto do médico quanto do paciente em realizar a contrarreferência, visto que para o médico, muitas vezes, o acompanhamento na UBS não será realizado com qualidade e, para o paciente, a preferência é de manter o atendimento com o especialista. Dessa forma, os pacientes acabam tendo mais retornos com o especialista de maneira a preencher as agendas do HRHC e aumentar a dificuldade de que novos pacientes sejam atendidos, o que sobrecarrega ainda mais a fila de espera dos pacientes encaminhados pela UBS.

Assim, para melhor análise da situação atual da fila de encaminhamentos para a angiologia, de forma a possibilitar um planejamento estratégico para sua redução e otimização do atendimento médico com o especialista, faz-se necessário a realização de uma descrição analítica e causal das filas tanto da UBS estudada nesse artigo quanto do HRHC de referência dela. Para isso, foram utilizadas duas ferramentas de análise, a teoria das filas e a simulação, as quais serão explanadas mais a frente.

\section{METODOLOGIA}




\section{Teoria das filas}

A teoria das filas é um método analítico, que por meio de fórmulas matemáticas, demonstra o comportamento de um sistema que ofereça serviço com demanda crescente e aleatória, permitindo dimensionamento, análise de viabilidade econômica e intervenção do serviço (3). Diferentemente de outras ferramentas de pesquisa operacional, a teoria das filas não é uma ferramenta de otimização; apenas determina a situação da fila atual, segundo análises matemáticas precisas e propriedades mensuráveis da fila, tais como, taxa de chegada, taxa de serviço, tempo de espera, capacidade do sistema, entre outros (4). Seu uso possibilita melhor compreensão da problemática atual para gerar melhorias. Além disso, facilita a verificação do comportamento de sistemas reais em situação aleatória (5).

No estudo em questão, há uma fila de espera com 4579 pacientes que aguardam atendimento médico com a especialidade de angiologia no HRHC analisado. Há um desbalanço entre demanda e capacidade de serviço, causando o surgimento da fila. O HRHC em questão recebe encaminhamentos médicos de diversas UBS e assim, toda a demanda proveniente dessas Unidades de Atenção Primária deve ser absorvida pelos angiologistas desse estabelecimento executante. Como é de se supor, as taxas de encaminhamentos à fila da angiologia não são constantes quando analisadas cada Unidade de Atenção Primária, havendo assim uma taxa de chegada de pacientes às UBS e uma taxa de encaminhamento $(\lambda)$ ao especialista. Da mesma forma, a taxa de serviço, ou seja, o tempo que demora para cada paciente ser agendado com o angiologista também pode variar, a depender da agenda médica. Dessa forma, o sistema de fila da angiologia para o HRHC, poderia ser processado, simplificadamente, como mostra a Figura 1 abaixo.

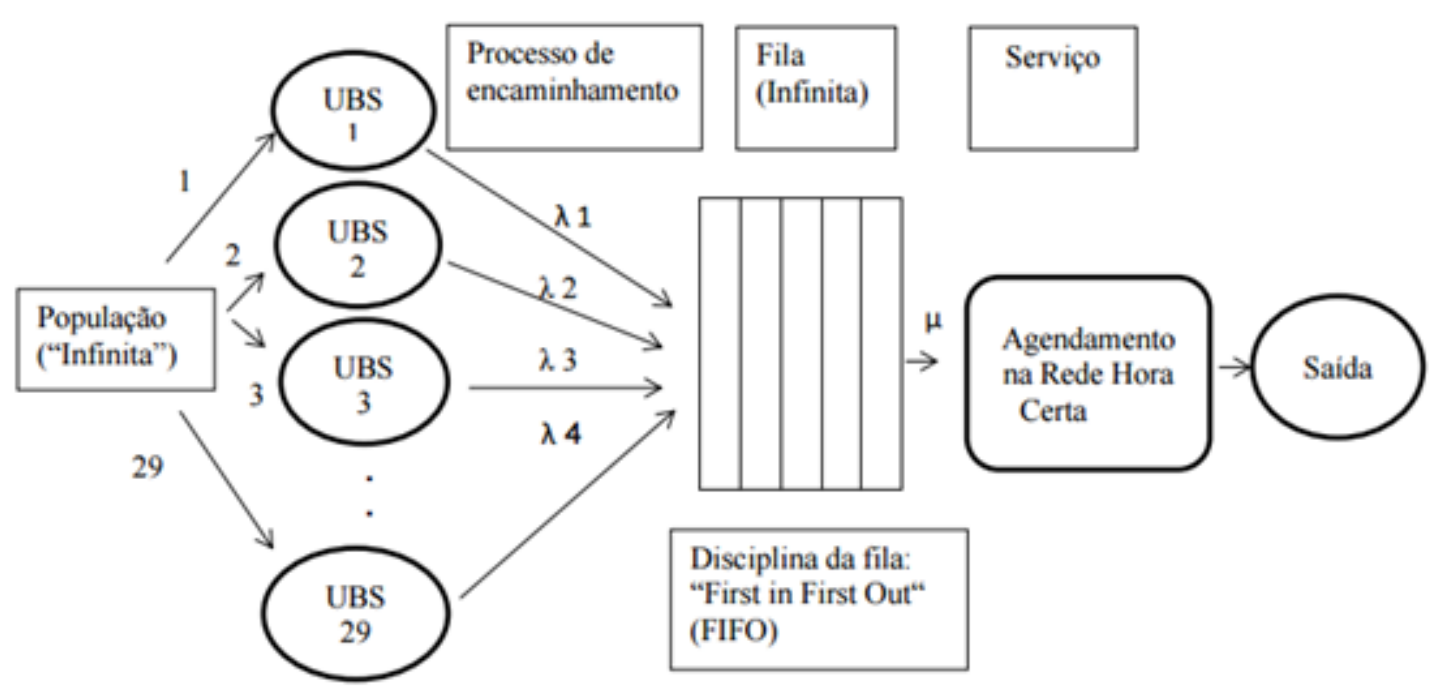

Figura 1. Esquema de formação da fila para angiologia no HRHC analisado. 
Assim, o modelo matemático usado para análise da situação atual da fila da angiologia no HRHC leva em consideração a taxa de chegada dos pacientes no HRHC ( $\lambda$ - encaminhamento para a fila feito pelas UBS), a taxa de serviço ( $\mu$ - tempo para agendamento no $\mathrm{HRHC}$ ), a quantidade de médicos angiologistas e o tamanho do sistema da fila. No caso específico desse projeto, é importante ressaltar que levaremos em consideração apenas um HRHC, situado na cidade de São Paulo. Em relação à capacidade do sistema de fila e a população de clientes, ambos serão considerados como "infinitos", haja vista a particularidade desse sistema: apesar dos pacientes das UBS terem que respeitar a regionalização, ou seja, devem residir na área de abrangência específica de atendimento do centro especializado em questão para que tenham direito de acesso ao serviço, o sistema aqui referido é aberto e para fins matemáticos, considerado "infinito"; a fila de espera não se configura como sistema físico, já que o paciente, após encaminhado a essa fila, aguarda contato telefônico das Unidades de Saúde para aviso do agendamento com o especialista (saída da fila).

\section{Simulação}

Outra ferramenta de análise matemática que promove uma melhor compreensão sobre o sistema em questão é a simulação. Se o problema em estudo for complexo, dinâmico e apresentar aleatoriedade, a simulação é uma ferramenta analítica de grande valia (6).

Um modelo matemático construído pela teoria das filas é composto por fórmulas que, via de regra, fornecem soluções mais rápidas do que a abordagem da simulação. Por outro lado, a teoria das filas impõe hipóteses simplificadoras para sistemas complexos, e como dito anteriormente, ela apenas determina a situação atual da fila. Quanto mais dinâmico e aleatório for um problema, maior será a aplicabilidade das ferramentas de simulação (6). O modelo de simulação visa capturar o comportamento de um dado sistema e representá-lo como um modelo computacional.

Para tal, deve haver uma minuciosa etapa de observação do fenômeno além da coleta de dados precisos, de forma a simular o sistema real o mais representativo possível (7). No caso desse projeto, os dados foram obtidos através de duas fontes: sistema SIGA Saúde e entrevistas com os gestores tanto da UBS quanto do HRHC analisados.

Após estudo da situação atual da fila e cálculo dos principais dados de análise, procuramos modelar o sistema através da simulação de forma a prever o comportamento probabilístico da fila. Assim, elencamos as variáveis de maior impacto na formação da fila e criamos planos de ação para otimizar o sistema. O software de simulação utilizado foi o SIMUL8 Professional.

\section{Modelagem dos dados de entrada: tratamento e escolha da distribuição probabilística}


A coleta de dados deve ser minuciosa e a mais precisa possível a fim de reproduzir o sistema de forma fiel. Deve-se levar em consideração quais são as variáveis de entrada da simulação (input) e quais serão utilizadas para validação do modelo. Dessa forma, para que essa metodologia seja confiável e passível de gerar resultados que possam ser utilizados no auxílio do planejamento estratégico, os dados gerados por ela devem ser compatíveis com os dados reais observados no fenômeno.

Assim, para melhor compreensão do cenário como um todo, coletamos e calculamos os dados referentes ao tamanho da fila atual para angiologia no $\mathrm{HRHC}$ analisado, a agenda médica dos especialistas desse estabelecimento, os encaminhamentos das Unidades de Atenção Primária para fila de "Primeira vez" com a angiologia e a taxa de agendamento no HRHC.

Durante o estudo de simulação, além da coleta de dados, precisamos nos ater ao tratamento e nos testes de aderência, os quais, são fundamentais para a melhor inferência das propriedades de um dado fenômeno aleatório. Assim, após a etapa de coleta de informações, denominada "amostragem", devemos modelar os dados de entrada. Para realizar a modelagem dos dados temos que obter os modelos probabilísticos que melhor representam os fenômenos que compõe o sistema em estudo.

No caso do atual projeto, temos como dados de entrada, a taxa entre chegadas de pacientes (número de encaminhamentos médicos para angiologia realizada pelas UBS dentro do período analisado), o tempo de atendimento (número de pacientes agendados para consulta com a angiologia no período analisado e a capacidade instalada da agenda médica dessa especialidade) e a fila inicial do serviço de angiologia no HRHC analisado (start up). Foram considerados como fenômenos aleatórios os encaminhamentos das Unidades de Atenção Primária e os agendamentos com os especialistas. No entanto, antes de utilizar uma distribuição estatística conhecida para prever o fenômeno, nos baseamos na série histórica (trace file) para modelar e validar a formação da fila atual. Assim, a partir dos dados coletados durante o período de 01/01/2017 a 23/06/2017, modelamos a formação da fila e através do software SIMUL8 Professional validamos os dados de entrada, conforme pode ser observado na Tabela 1. A partir de então, realizamos a análise da melhor distribuição probabilística para o caso. Utilizando algumas ferramentas da estatística descritiva, pudemos explorar melhor o nosso conjunto de dados dos fenômenos aleatórios. Assim, extraímos as medidas de posição (média e mediana) e de dispersão (desvio padrão).

Tabela 1. Medidas de posição e de dispersão dos dados de entrada.

\begin{tabular}{|l|r|r|}
\hline Fenômenos aleatórios & $\begin{array}{l}\text { Encaminhamentos para } \\
\text { Angiologia (Entrada na fila) }\end{array}$ & Agendamentos (Saída da fila) \\
\hline Média (mensal) & 438 & 109 \\
\hline
\end{tabular}




\begin{tabular}{|l|r|r|}
\hline Mediana & 455 & 109,5 \\
\hline Desvio padrão & 89,26 & 23,32 \\
\hline
\end{tabular}

Como a coleta de dados foi feita através da série histórica, e esta é pautada em dados mensais, conseguimos os números de encaminhamentos por mês de cada Unidade de Atenção Primária e os agendamentos mensais de "Primeira vez" para angiologia no HRHC analisado. Dessa forma, nossa amostra (n) tanto para encaminhamento, quanto para agendamento, era $n=6$ (6 meses de análise). Para escolher o melhor modelo probabilístico podem ser usados softwares de ajuste de dados (fitting). No nosso caso, usamos o Stat Fit ferramenta atrelada ao SIMUL8 Professional. No entanto, como o " $n$ " obtido foi muito baixo, a análise pelos testes estatísticos ficou prejudicada. Assim, a distribuição escolhida foi o "Average", tanto para a taxa de entrada (encaminhamentos) quanto para a taxa de serviço (agendamentos). $O$ "Average" pressupõe uma distribuição normal com desvio padrão de $25 \%$ em relação à média (6). Dessa forma, para usar a distribuição probabilística "Average" nas simulações, partimos do pressuposto de que não haveriam alterações significativas nos dados de entrada nos próximos 18 meses. Assim, pudemos validar os dados de entrada dentro do período analisado usando o SIMUL8 Professional. Os parâmetros calculados e utilizados para validação do modelo de simulação estão listados na Tabela 2.

Tabela 2. Parâmetros de entrada calculados a partir dos dados históricos da UBS e do HRHC analisados.

\begin{tabular}{|l|r|}
\hline SIMUL8 & Parâmetros (Input) \\
\hline Tempo de atendimento (agendamentos) & 16,1 minutos \\
\hline Fila Inicial (01/01/2017) & 2603 pacientes \\
\hline Tempo entre chegadas (encaminhamentos) & 94,71 minutos \\
& \\
\hline
\end{tabular}

Como resultado da simulação feita em um intervalo de 6 meses, o software gerou dados muito próximos com o encontrado na realidade (parâmetros de 23/06/2017), conforme mostra a Tabela 3.

Tabela 3. Resultados obtidos com o software após simulação com os dados modelados e os números reais usados como base de validação. 


\begin{tabular}{|l|r|r|}
\hline $\begin{array}{l}\text { Intervalo de simulação } \\
\text { (01/01/2017 até } \\
\text { 23/06/2017) }\end{array}$ & Resultados SIMUL8 (Output) & $\begin{array}{l}\text { Realidade atual } \\
\text { (23/06/2017) }\end{array}$ \\
\hline Encaminhamentos & 2620 & 2630 \\
\hline Fila Final & 4546 & 4579 \\
\hline Agendamentos & 676 & 654 \\
\hline
\end{tabular}

Dessa forma, como mostra a Tabela 4, as porcentagens de erros obtidas foram baixas.

Tabela 4. Comparação entre os resultados obtidos através da simulação com os dados reais.

\begin{tabular}{|l|r|r|}
\hline & $\begin{array}{l}\text { Diferença dos resultados em } \\
\text { número absoluto }\end{array}$ & Porcentagem \\
\hline Encaminhamentos & 10 & $0,4 \%$ \\
\hline Fila final & 33 & $0,7 \%$ \\
\hline Agendamentos & -22 & $3,3 \%$ \\
\hline
\end{tabular}

Como a porcentagem de erros foi abaixo de $5 \%$, os dados de entrada foram considerados aptos a serem utilizados para as simulações (6).

Para efeito de cálculo, outro parâmetro de entrada importante para levarmos em consideração é o tempo de agenda dos angiologistas destinado para consulta médica de "Primeira vez". Na agenda desses especialistas, há tanto pacientes de "Primeira vez" quanto de "Retorno", porém apenas os agendamentos do primeiro grupo impactam na fila analisada nesse estudo, já que a fila para "Retorno" é outra. Assim, através dos dados obtidos pelo sistema SIGA saúde, pudemos calcular o tempo semanal de agenda destinado para consultas de angiologia de "Primeira vez", sendo este de 8,19 horas. No $\mathrm{HRHC}$ analisado há 3 médicos angiologistas contratados que trabalham de segunda à quinta-feira, variando os períodos de agenda em cada dia. Como o tempo semanal destinado é de 8,19 horas e há 4 dias disponíveis para tal agenda, temos que o tempo médio diário é de 2,04 horas.

\section{RESULTADOS}


Visto que o sistema se encontra instável, testamos através da técnica da simulação probabilística com o SIMUL8 Professional diversas situações dos parâmetros do problema buscando otimizar a operação do fenômeno em questão. Assim, dividimos as simulações em 3 (três) macro situações:

1. Prospecção futura (como o sistema de formação de filas irá prosseguir caso os parâmetros se mantenham inalterados).

2. Diminuição da taxa entre chegadas de pacientes (encaminhamentos para o $\mathrm{HRHC}$ ).

3. Aumento da taxa de atendimento (agendamentos com o especialista).

A primeira situação avaliada foi a de inalterabilidade dos parâmetros.

Calculamos então qual seria a fila de pacientes para angiologia no HRHC analisado no período de 6 (seis) meses a partir de 23/06/2017 (data de realização do presente estudo). Para tal, usamos a taxa entre chegadas de 94,71 minutos (vide Tabela 2), o tempo médio de atendimento de 16,1 minutos (vide Tabela 2), fila inicial de 4579 (vide Tabela 3) e tempo médio de trabalho de 2 horas e 04 minutos. Conforme mostra a tabela 5, caso os parâmetros não se alterem, em 6 meses, a fila para angiologia passaria de 4579 para 6384 pacientes de "Primeira vez".

Tabela 5. Prospecção futura

\begin{tabular}{|l|l|l|l|l|l|}
\hline Parâmetros & $\begin{array}{l}\text { Tempo médio } \\
\text { atendimento } \\
\text { (minutos) }\end{array}$ & $\begin{array}{l}\text { Tempo médio } \\
\text { entre chegadas } \\
\text { (minutos) }\end{array}$ & $\begin{array}{l}\text { Tempo de } \\
\text { agenda dos } \\
\text { médicos por } \\
\text { dia }\end{array}$ & $\begin{array}{l}\text { Fila } \\
\text { início }\end{array}$ & $\begin{array}{l}\text { Tempo de } \\
\text { simulação }\end{array}$ \\
\hline & 16,1 & 94,71 & 2 h04 & 4579 & 6 meses \\
\hline $\begin{array}{l}\text { Resultados } \\
\text { SIMUL8 }\end{array}$ & $\begin{array}{l}\text { Encaminhamen } \\
\text { tos }\end{array}$ & Fila final & Atendimentos & & \\
\hline & 2543 & 6384 & 737 & & \\
\hline
\end{tabular}

No caso da diminuição da taxa entre chegadas de pacientes (encaminhamentos ao HRHC), atuamos sob duas perspectivas: redução dos encaminhamentos de CIDs (Classificação Internacional de Doenças) considerados discutíveis, ou seja, que poderiam ser reavaliados quanto a sua correta indicação à especialidade angiologia (cerca de 5\%) e diminuição dos encaminhamentos de varizes não complicadas que poderiam ser acompanhadas na própria UBS e representam cerca de $60 \%$ dos encaminhamentos gerais para angiologia do HRHC analisado. Após simulação da situação com $5 \%$ de redução dos encaminhamentos (CIDs discutíveis), a fila final seria de 6263 pacientes após 6 meses, conforme mostra a Tabela 6. A 
redução de $5 \%$ do número de encaminhamentos altera o tempo médio entre chegadas de 94,71 minutos para 99,59 minutos.

Tabela 6. Simulação com redução dos encaminhamentos de CIDs considerados discutíveis (5\%).

\begin{tabular}{|l|l|l|l|l|l|}
\hline Parâmetros & $\begin{array}{l}\text { Tempo médio } \\
\text { atendimento } \\
\text { (minutos) }\end{array}$ & $\begin{array}{l}\text { Tempo médio } \\
\text { entre chegadas } \\
\text { (minutos) }\end{array}$ & $\begin{array}{l}\text { Tempo de } \\
\text { agenda dos } \\
\text { médicos por } \\
\text { dia }\end{array}$ & $\begin{array}{l}\text { Fila } \\
\text { início }\end{array}$ & $\begin{array}{l}\text { Tempo de } \\
\text { simulação }\end{array}$ \\
\hline & 16,1 & 99,59 & 2 h04 & 4579 & 6 meses \\
\hline $\begin{array}{l}\text { Resultados } \\
\text { SIMUL8 }\end{array}$ & Encaminhamentos & Fila final & Atendimentos & & \\
\hline & 2422 & 6263 & 737 & & \\
\hline
\end{tabular}

Assim, a redução dos encaminhamentos de CIDs discutíveis teria um impacto muito pequeno na fila de espera. Na situação de redução de $60 \%$ dos encaminhamentos referentes aos casos de varizes não complicadas, simulamos como teria ficado a fila de espera em $23 / 06 / 2017$ caso essa ação fosse aprovada no início do período analisado, 01/01/2017. Observando os resultados do software na Tabela 7 , podemos concluir que mesmo que $60 \%$ do total dos encaminhamentos fossem reduzidos, a fila de espera ainda teria aumentado. No entanto, se comparado com a situação real, ou seja, mantendo os encaminhamentos das varizes não complicadas, a fila teria crescido $34 \%$ a menos.

Tabela 7. Simulação com redução dos encaminhamentos dos CIDs de Varizes não Complicadas (60\%).

\begin{tabular}{|l|l|l|l|l|l|}
\hline Parâmetros & $\begin{array}{l}\text { Tempo médio } \\
\text { atendimento } \\
\text { (minutos) }\end{array}$ & $\begin{array}{l}\text { Tempo médio } \\
\text { entre chegadas } \\
\text { (minutos) }\end{array}$ & $\begin{array}{l}\text { Tempo de } \\
\text { agenda dos } \\
\text { médicos por } \\
\text { dia }\end{array}$ & $\begin{array}{l}\text { Fila } \\
\text { início }\end{array}$ & $\begin{array}{l}\text { Tempo de } \\
\text { simulação }\end{array}$ \\
\hline $\begin{array}{l}\text { Resultados } \\
\text { SIMUL8 }\end{array}$ & Encaminhamentos & Fila Final & Atendimentos & & 173 dias \\
\hline & 1047 & 236,71 & 676 & & \\
\hline
\end{tabular}


Para o caso de aumento da taxa de atendimento, foram consideradas as seguintes situações: acréscimo de 24 horas por semana de atendimento médico apenas para realizar consultas de "Primeira vez" (Tabela 8); acréscimo de 24 horas por semana de atendimento médico e redução de $5 \%$ do tempo entre chegadas devido aos encaminhamentos com CIDs discutíveis (Tabela 9); acréscimo de 30 horas por semana de atendimento médico (Tabela 10); acréscimo de 30 horas por semana de atendimento médico e diminuição do tempo de atendimento de 16,1 minutos por paciente para 15 minutos (Tabela 11); acréscimo de 24 horas por semana de atendimento médico e redução de $60 \%$ dos encaminhamentos de varizes não complicadas (Tabela 12); acréscimo de 36 horas por semana de atendimento médico destinado para atendimentos de "Primeira vez" (Tabela 13). Foi descontada 1 hora de almoço para cada 12 horas de plantão semanal acrescentado. Após as simulações, as situações descritas ficaram assim definidas.

Tabela 8. Acréscimo de 24 horas por semana de médico angiologista.

\begin{tabular}{|l|r|r|r|r|r|}
\hline Parâmetros & $\begin{array}{l}\text { Tempo médio } \\
\text { atendimento } \\
\text { (minutos) }\end{array}$ & $\begin{array}{l}\text { Tempo médio } \\
\text { entre chegadas } \\
\text { (minutos) }\end{array}$ & $\begin{array}{l}\text { Tempo de } \\
\text { agenda dos } \\
\text { médicos por } \\
\text { dia }\end{array}$ & $\begin{array}{l}\text { Fila } \\
\text { início }\end{array}$ & $\begin{array}{l}\text { Tempo de } \\
\text { simulação }\end{array}$ \\
\hline 16,1 & 94,71 & $7 \mathrm{~h} 34$ & 4579 & 2 anos \\
\hline $\begin{array}{l}\text { Resultados } \\
\text { SIMUL8 }\end{array}$ & Encaminhamentos & Fila final & Atendimentos & & \\
\hline & 11090 & 3879 & 11789 & & \\
\hline
\end{tabular}

Tabela 9. Acréscimo de 24 horas por semana de médico angiologista e redução de $5 \%$ dos encaminhamentos devido a CIDs discutíveis.

\begin{tabular}{|l|r|l|l|l|r|}
\hline Parâmetros & $\begin{array}{l}\text { Tempo médio } \\
\text { atendimento } \\
\text { (minutos) }\end{array}$ & $\begin{array}{l}\text { Tempo médio } \\
\text { entre chegadas } \\
\text { (minutos) }\end{array}$ & $\begin{array}{l}\text { Tempo de } \\
\text { agenda dos } \\
\text { médicos por } \\
\text { dia }\end{array}$ & $\begin{array}{l}\text { Fila } \\
\text { início }\end{array}$ & $\begin{array}{l}\text { Tempo de } \\
\text { simulação }\end{array}$ \\
\hline & 16,1 & 99,59 & $7 \mathrm{~h} 34$ & 4579 & 2 anos \\
\hline $\begin{array}{l}\text { Resultados } \\
\text { SIMUL8 }\end{array}$ & Encaminhamentos & Fila final & Atendimentos & & \\
\hline & 10539 & 3328 & 11789 & & \\
\hline
\end{tabular}

Tabela 10. Acréscimo de 30 horas por semana de médico angiologista. 


\begin{tabular}{|l|r|r|r|r|r|}
\hline Parâmetros & $\begin{array}{l}\text { Tempo médio } \\
\text { atendimento } \\
\text { (minutos) }\end{array}$ & $\begin{array}{l}\text { Tempo médio } \\
\text { entre chegadas } \\
\text { (minutos) }\end{array}$ & $\begin{array}{l}\text { Tempo de } \\
\text { agenda dos } \\
\text { médicos por } \\
\text { dia }\end{array}$ & $\begin{array}{l}\text { Fila } \\
\text { início }\end{array}$ & $\begin{array}{l}\text { Tempo de } \\
\text { simulação }\end{array}$ \\
\hline & 16,1 & 94,71 & $9 \mathrm{h04}$ & 4579 & 2 anos \\
\hline $\begin{array}{l}\text { Resultados } \\
\text { SIMUL8 }\end{array}$ & Encaminhamentos & Fila final & Atendimentos & & \\
\hline & 11090 & 1539 & 14129 & & \\
\hline
\end{tabular}

Tabela 11. Acréscimo de 30 horas por semana de médico angiologista e redução do tempo médio de atendimento para 15 minutos.

\begin{tabular}{|l|l|l|l|l|l|}
\hline Parâmetros & $\begin{array}{l}\text { Tempo médio } \\
\text { atendimento } \\
\text { (minutos) }\end{array}$ & $\begin{array}{l}\text { Tempo médio } \\
\text { entre chegadas } \\
\text { (minutos) }\end{array}$ & $\begin{array}{l}\text { Tempo de } \\
\text { agenda dos } \\
\text { médicos por } \\
\text { dia }\end{array}$ & $\begin{array}{l}\text { Fila } \\
\text { início }\end{array}$ & $\begin{array}{l}\text { Tempo de } \\
\text { simulação }\end{array}$ \\
\hline 15 & 94,71 & $9 h 04$ & 4579 & 2 anos \\
\hline $\begin{array}{l}\text { Resultados } \\
\text { SIMUL8 }\end{array}$ & Encaminhamentos & Fila final & Atendimentos & & \\
\hline & 11090 & 500 & 15168 & & \\
\hline
\end{tabular}

Tabela 12. Acréscimo de 24 horas por semana de médico angiologista e redução de $60 \%$ dos encaminhamentos devido a varizes não complicadas.

\begin{tabular}{|l|l|l|l|r|r|}
\hline Parâmetros & $\begin{array}{l}\text { Tempo médio } \\
\text { atendimento } \\
\text { (minutos) }\end{array}$ & $\begin{array}{l}\text { Tempo médio } \\
\text { entre chegadas } \\
\text { (minutos) }\end{array}$ & $\begin{array}{l}\text { Tempo de } \\
\text { agenda dos } \\
\text { médicos por } \\
\text { dia }\end{array}$ & $\begin{array}{l}\text { Fila } \\
\text { início }\end{array}$ & $\begin{array}{l}\text { Tempo de } \\
\text { simulação }\end{array}$ \\
\hline & 16,1 & 236,71 & 7 h34 & 4579 & 1,3 anos \\
\hline $\begin{array}{l}\text { Resultados } \\
\text { SIMUL8 }\end{array}$ & Encaminhamentos & Fila final & Atendimentos & & \\
\hline & 2875 & 14 & 7440 & & \\
\hline
\end{tabular}

Tabela 13. Acréscimo de 36 horas por semana de médico angiologista. 


\begin{tabular}{|l|l|l|l|r|r|}
\hline Parâmetros & $\begin{array}{l}\text { Tempo médio } \\
\text { atendimento } \\
\text { (minutos) }\end{array}$ & $\begin{array}{l}\text { Tempo médio } \\
\text { entre chegadas } \\
\text { (minutos) }\end{array}$ & $\begin{array}{l}\text { Tempo de } \\
\text { agenda dos } \\
\text { médicos por } \\
\text { dia }\end{array}$ & $\begin{array}{l}\text { Fila } \\
\text { início }\end{array}$ & $\begin{array}{l}\text { Tempo de } \\
\text { simulação }\end{array}$ \\
\hline & 16,1 & 94,71 & $10 h 19$ & 4579 & 2 anos \\
\hline $\begin{array}{l}\text { Resultados } \\
\text { SIMUL8 }\end{array}$ & Encaminhamentos & Fila final & Atendimentos & & \\
\hline & 11090 & 10 & 15659 & & \\
\hline
\end{tabular}

\section{CONCLUSÃO}

Dentre todas as situações simuladas, as que geram maiores impactos na redução da fila são aquelas que envolvem acréscimo em horas semanais de atendimento médico exclusivo para "Primeira vez", ou seja, que aumentam a taxa de atendimento realizado pelos angiologistas. Pelos casos simulados, para reduzir a fila de espera da angiologia para 10 pacientes em 2 anos, seriam necessárias 36 horas a mais de atendimento médico por semana, o que geraria um custo baixo frente ao impacto que traria tal ação. A problemática das filas de atendimento especializado no SUS é uma prioridade de saúde pública, e através da simulação podemos prever o comportamento do sistema de formação das filas e atuar nos pontos de maior impacto, de forma assertiva e mais eficiente. Outra alternativa, seria de implementar estratégias de redução dos encaminhamentos, no entanto, só trariam resultados eficientes se tais medidas fossem agregadas ao aumento da taxa de atendimento médico. Assim, uma possibilidade para redução da fila de angiologia no $\mathrm{HRHC}$ analisado seria de reduzir os encaminhamentos de varizes não complicadas, correspondentes a cerca de $60 \%$ do total dos encaminhamentos e acrescentar 24 horas de plantão semanal de médico angiologista. Esta última alternativa levaria 1,3 anos para reduzir a fila de espera para 14 pacientes.

Após todas as análises simuladas, foram elaborados planos de ação que visam otimizar o processo do fenômeno em questão, principalmente no que se refere às alternativas assistenciais de redução dos encaminhamentos de pacientes para a angiologia, que em conjunto com a contratação de novos profissionais médicos, pode ser uma solução para a questão das longas filas de espera por atendimento.

Os planos de ação estão ordenados pelo impacto que apresentam frente às questões levantadas no projeto e na ordem cronológica sequencial para que os planos, caso sejam acatados, ocorram de forma eficiente.

\section{Plano de ação 1: Manejo de varizes não complicadas na UBS}


Matriz esforço x impacto: Alto esforço e alto impacto.

Após nossa observação de que $81,5 \%$ dos encaminhamentos da UBS analisada para a angiologia do HRHC de sua referência são de varizes de Membros Inferiores e 63\% são varizes não complicadas, propomos que haja uma organização da UBS a fim de que os casos de varizes que não tenham complicação sejam acompanhados clinicamente pelos médicos na própria UBS, em vez de encaminhar esse paciente para a fila de agendamento da angiologia, reduzindo a quantidade de encaminhamentos desse tipo e priorizando casos mais graves e que precisam mais urgentemente de avaliação com o especialista. Para tal, pode ser utilizada a escala CEAP, escala consagrada na literatura médica em angiologia (9) para classificação de varizes de membros inferiores. Basicamente é uma escala de $\mathrm{C} 0$ a $\mathrm{C} 6$, classificada conforme características da patologia. Por exemplo, $\mathrm{C} 0$ corresponderia a "sem sinais visíveis ou palpáveis de doença venosa" e C6 "alterações tróficas com úlcera aberta".

Segundo a literatura médica (9), os estágios $\mathrm{C} 0$ a C2 podem ser manejados clinicamente na UBS através de controle de sintomas com medicações analgésicas para dor, uso de meias elásticas, decúbito (ficar deitado) com elevação de pernas principalmente ao fim da tarde e a noite, exercícios regulares (como caminhada) e perda de peso. Há a possibilidade de prescrever-se flebotônicos, ou seja, medicamentos que ajudam na circulação das veias de membros inferiores, como a diosmina, para redução de sintomas. Entretanto, esses medicamentos não são disponíveis no SUS e não mudam a evolução da doença, melhorando apenas a sintomatologia que as varizes causam no paciente (10). No estágio C3, no qual já há edema (inchaço) nos pés, se o paciente responder bem ao tratamento clínico citado, ele pode ser acompanhado na UBS. Se não responder bem ao tratamento, ocorrendo manutenção da dor ou outros sintomas, deve ser encaminhado para o angiologista, assim como os estágios C4 a C6 devem ser encaminhados diretamente ao especialista.

Dessa forma, uma proposta seria que fosse implantado um grupo de educação continuada para os médicos da UBS, com enfoque em manejo clínico de pacientes com varizes, a fim de qualificar melhor os encaminhamentos para 0 angiologista do HRHC, uma vez que as filas são longas e as vagas menos disponíveis.

\section{Plano de ação 2: Mudança no protocolo de encaminhamento de varizes de Membros Inferiores para o HRHC}

Matriz impacto x esforço: Alto esforço e médio impacto.

Verificamos que o atual protocolo da Secretaria Municipal de Saúde em conjunto com os Hospitais da Rede Hora Certa para encaminhamento de varizes de membros inferiores é pouco específico no quesito de quando priorizar o encaminhamento de varizes para o angiologista. A priorização do 
caso é definida por "Grau de sintomatologia" e "estágio do quadro", sem especificar com mais detalhes. Isso abre margem para interpretação e possibilidade de encaminhamento de pacientes que provavelmente poderiam ser acompanhados clinicamente pelos médicos na UBS. Dessa forma, sugerimos um refinamento do protocolo, baseado na escala CEAP, conforme citado no item anterior, definindo melhor os motivos e prioridades de encaminhamento. Uma sugestão, por exemplo, seria o acompanhamento de varizes Classe C0 a C3 (essa última com boa resposta ao tratamento clínico) pelos médicos da UBS e de C3 com pouca resposta a tratamento clínico até C6 seria sugerido o encaminhamento para o angiologista. Tal ação ajudaria a otimizar o fluxo de encaminhamentos para o HRHC nessa especialidade, priorizando os casos mais graves.

Plano alternativo: Médio esforço x alto impacto.

Uma vez que tempo é essencial nesse caso, sugerimos a criação de uma Orientação de encaminhamento de Varizes de membros inferiores com complicação para a angiologia do $\mathrm{HRHC}$ e manejo das não complicadas na UBS, conforme sugerido no Plano de Ação 1 (baseando-se no CEAP). Para tal, além da orientação aos médicos sobre o manejo de varizes na UBS, sugerimos deixar nos consultórios da UBS em fácil acesso para os médicos a escala CEAP, tanto escrita quanto visual, ajudando-o a realizar o diagnóstico correto de varizes e a manejá-las da forma mais adequada, acompanhando as que forem possíveis e encaminhando as que forem necessárias. A escala poderia estar disponível, por exemplo, afixada na mesa de atendimento do médico, na parede mais próxima dele no consultório ou no desktop do computador. $\mathrm{O}$ importante é ser visível, clara e de fácil acesso.

\section{Plano de ação 3: Requalificação da fila de agendamento para a angiologia do HRHC}

Matriz esforço x impacto: Alto esforço e médio impacto.

Devido à fila atual de 195 pacientes para a angiologia do $\mathrm{HRHC}$ analisado e o tempo de espera de quase 10 meses para conseguir agendamento nesse especialista, sugerimos uma requalificação da fila de espera das UBS. Para tal, seria necessária uma reavaliação dos prontuários dos pacientes da fila para estratificar o grau de urgência dos encaminhamentos e reconvocar os que forem necessários para reavaliação médica. Dessa forma, poderia ser definida a priorização ou não do encaminhamento perante a fila. Propomos essa ideia, pois, apesar de haver um sistema de priorização da fila no pedido de encaminhamento, que pode ter a prioridade classificada como alta, média ou baixa, na prática, a maioria deles é classificada como alta, mesmo não sendo um caso grave. Assim, nessa fila de 10 meses podemos ter casos de pacientes com varizes simples sem complicação concorrendo com outros com varizes complicadas com ulcerações ou com pé diabético com feridas de difícil cicatrização, que precisariam de avaliação com o angiologista com mais prioridade. 
Acreditamos que uma requalificação da fila ajudaria a otimizar os encaminhamentos e evitar complicações para os pacientes mais graves, encurtando o tempo de espera para consulta com o angiologista.

\section{Plano de ação 4: Análise e entendimento de CIDs de encaminhamento e agendamento avaliados como discutíveis}

Matriz esforço x impacto: Alto esforço e baixo impacto.

Após nossa análise, tanto os encaminhamentos realizados pelas UBS para a angiologia do $\mathrm{HRHC}$, quanto os agendamentos realizados por esse último estabelecimento, verificamos que existem CIDs considerados discutíveis para o contexto de angiologia. Ressaltamos que esses CIDs, além de terem um grande potencial de serem encaminhamentos incorretos que irão ocupar espaço na agenda dos especialistas, atrapalham as análises de cenário, principalmente quando há CIDs visivelmente fora de contexto (Exemplo: anencefalia encaminhada para angiologia). Dessa forma, consideramos que seja importante que as UBS verifiquem aqueles considerados discutíveis em seus encaminhamentos, e o próprio HRHC também verificar isso em seus agendamentos de primeira consulta e retorno. Com CIDs mais precisos, será possível análises futuras mais claras e com menos fatores de confusão. Essa ação demandaria verificação dos CIDs nas tabelas de encaminhamento das UBSs e agendamentos na angiologia do HRHC e o levantamento dos prontuários dos pacientes com esses CIDs e verificação do que houve exatamente caso a caso para que houvesse o desvio do CID.

\section{Agradecimentos}

Aos coordenadores e funcionários da Associação Saúde da Família (ASF) que cooperaram na discussão da proposta e facilitaram a implementação do projeto disponibilizando dados e infraestrutura de apoio.

\section{Referências}

1) Azevedo, ALM; Costa, AM. La estrecha puerta de entrada del Sistema Único de Salud (SUS): una valoración del acceso en la Estrategia de Salud de la Familia (ESF). Interface - Comunic., Saude, Educ; 2010.

2) World Health Organization. Primary Health Care. Genebra, WHO, 1978.

3) Arenales, MN. Pesquisa Operacional. $6^{\circ}$ ed. Rio de Janeiro; Elsevier, 2007. 524p.

4) Aurelio, M. Teoria das filas e das simulações (apostila). Santo André; UNIA, 2004. 
5) Chaves, ALF; et al. Estudo da teoria das filas em um Sistema médicohospitalar na cidade de Belém-PA. 2011. Disponível em:

http://www.abepro.org.br/biblioteca/enegp2011_TN_STP_140_880.

Acesso em: 24/06/2017.

6) Chwif, L; Medina, AC. Modelagem e simulação de eventos discretos. $4^{\circ}$ ed. Rio de Janeiro; Elsevier, 2015.

7) Mann, L; Taylor, KF. Queue counting: the effect motives upon estimates of numbers in waiting lines. J Pers Soc Psychol, USA, 1969 Jun; 12(2); 95-103.

8) Teixeira, MM. Introdução à Teoria Das Filas. Disponível em: https://www.ft.unicamp.br/ marlih/ST565/intro-filas.pdf. Acesso em: 26/06/2017.

9) Ragazzo, L; Fernandes AR. Principais temas em Cirurgia Vascular e Cirurgia Pediátrica para residência médica. $1^{\circ}$ ed. São Paulo: Medcel, 2014.

10)Santos, MC. Uso dos flebotônicos no tratamento da doença venosa crônica. Revista Brasileira de Medicina, Belo Horizonte, v. 68, n. 4, abr. 2011. Disponível em:

http://www.moreirajr.com.br/revistas.asp?fase=r003\&id_materia=4604. Acesso em: 10 jul. 2017.

Recebido: 06 de junho de 2018. Publicado: 16 de julho de 2018

Correspondência: Raphael Kaeriyama e Silva. E-mail:

raphaelsilva97@gmail.com

Conflito de interesses: os autores declararam não haver conflito de interesses.

(C) This is an Open Access article distributed under the terms of the Creative Commons Attribution License, which permits unrestricted use, distribution, and reproduction in any medium, provided the original work is properly cited 